\title{
Convergence rates to the damped system of compressible adiabatic flow through porous media with boundary effect
}

\section{Lina Zhang ${ }^{1}$, Shifeng Geng ${ }^{1 *}$ (i) and Yuling Gao'}

"Correspondence:

sfgeng@xtu.edu.cn

${ }^{1}$ School of Mathematics and

Computational Science, Xiangtan

University, Xiangtan, China

\begin{abstract}
In this paper, we consider convergence rates to solutions for the damped system of compressible adiabatic flow through porous media with boundary effect. Compared with the results obtained by Pan, the better convergence rates are obtained in this paper. Our approach is based on the technical time-weighted energy estimates.
\end{abstract}

Keywords: System of compressible adiabatic flow through porous media; Boundary effect; Damped; Convergence rates

\section{Introduction}

We investigate the following initial boundary problem for damped hyperbolic conservation laws:

$$
\left\{\begin{array}{l}
v_{t}-u_{x}=0, \quad(x, t) \in R^{+} \times R^{+}, \\
u_{t}+p(v, s)_{x}=-u, \\
s_{t}=0
\end{array}\right.
$$

which describes the motion of the compressible adiabatic flow through porous media. Here $u$ denotes the velocity, $v$ is the specific volume, $s$ stands for the entropy, $p$ is the pressure with $p_{v}(v, s)<0$ for $v>0$. For simplicity, we take $p(v, s)=(\gamma-1) v^{-\gamma} e^{s}$ with $\gamma>1$, which is the case for the polytropic gas.

We consider the initial value condition

$$
(v, u, s)(x, 0)=\left(v_{0}, u_{0}, s_{0}\right)(x) \rightarrow\left(v_{+}, u_{+}, s_{+}\right), \quad v_{+}>0, \text { as } x \rightarrow+\infty
$$

and the boundary condition

$$
v(0, t)=v_{0}(0)=v_{-}>0,
$$

(c) The Author(s) 2019. This article is licensed under a Creative Commons Attribution 4.0 International License, which permits use, sharing, adaptation, distribution and reproduction in any medium or format, as long as you give appropriate credit to the original author(s) and the source, provide a link to the Creative Commons licence, and indicate if changes were made. The images or other third party material in this article are included in the article's Creative Commons licence, unless indicated otherwise in a credit line to the material. If material is not included in the article's Creative Commons licence and your intended use is not permitted by statutory regulation or exceeds the permitted use, you will need to obtain permission directly from the copyright holder. To view a copy of this licence, visit http://creativecommons.org/licenses/by/4.0/. 
or

$$
u(0, t)=0
$$

It is known that the damping will create the diffusive phenomena of a hyperbolic wave, that is to say, the hyperbolic wave behaves like diffusive wave when $t$ tends to infinity. Pan [1] has proved that the solutions of these two problems (1.1)-(1.4) are captured by their related diffusive problems:

$$
\left\{\begin{array}{l}
\tilde{v}_{t}-\tilde{u}_{x}=0, \quad(x, t) \in R^{+} \times R^{+} \\
p(\tilde{v}, s)_{x}=-\tilde{u} \\
s_{t}=0 \\
(\tilde{v}, s)(x, 0)=\left(\tilde{v}_{0}, s_{0}\right)(x) \rightarrow\left(v_{+}, s_{+}\right), \quad v_{+}>0, \text { as } x \rightarrow+\infty \\
\tilde{v}(0, t)=\tilde{v}_{0}(0)=v_{-}
\end{array}\right.
$$

and

$$
\left\{\begin{array}{l}
\tilde{v}_{t}-\tilde{u}_{x}=0, \quad(x, t) \in R^{+} \times R^{+} \\
p(\tilde{v}, s)_{x}=-\tilde{u} \\
s_{t}=0 \\
(\tilde{v}, s)(x, 0)=\left(\tilde{v}_{0}, s_{0}\right)(x) \rightarrow\left(v_{+}, s_{+}\right), \quad v_{+}>0, \text { as } x \rightarrow+\infty \\
p(\tilde{v}, s)_{x}(0, t)=0
\end{array}\right.
$$

Zheng [2] proved the existence of global smooth solutions to the Cauchy problem (1.1) for small initial value. The asymptotic behavior of these solutions is considered for some specific initial value; see [3-5] and [6]. By choosing some particular initial value $\tilde{v}_{0}(x)$, Geng and Wang [7] proved that the best asymptotic profile of Eqs. (1.1) is a specific solution to the corresponding parabolic equation. Furthermore, the case for the general initial data has been investigated by Pan in [8] and the decay rates have been proved. By the characteristic analysis and the energy estimates, Hsiao and Pan [9] considered the initial boundary value problem for system (1.1). For the case of isentropic case where $s \equiv$ constant, Hsiao and Liu [10] first proved that the global smooth solution of the Cauchy problem (1.1) asymptotically converges to that of the corresponding parabolic equation and derived the convergence rate $\|(v-\tilde{v}, u-\tilde{u})(t)\|_{L^{\infty}}=O\left(t^{-1 / 2}, t^{-1 / 2}\right)$. The better convergence rates were obtained in [11-13]. For the other related interesting results of isentropic Euler equations, we refer to [14-22] and the references therein.

The main purpose of this paper is to use the technical time-weighted estimate and obtain the better convergence rates, which do not have $(1+\log (1+t))^{\beta_{1}}$ and $(1+\log (1+t))^{\beta_{2}}$ with $\beta_{1}>\frac{1}{3}, \beta_{2}>\frac{1}{2}$, in comparison with the results obtained by Pan in [1].

As in [1], in order to construct the asymptotic profiles for (1.1)-(1.3), we introduce the variables

$$
a(x)=(\gamma-1)^{-\frac{1}{\gamma}} e^{-\frac{1}{\gamma} s(x)}, \quad \text { and } \quad \omega \equiv a(x) \tilde{v}=p(\tilde{v}, s)^{-\frac{1}{\gamma}}
$$


then (1.5) is equivalent to the following problem:

$$
\left\{\begin{array}{l}
\omega_{t}+a(x)\left(\omega^{-\gamma}\right)_{x x}=0, \quad(x, t) \in R^{+} \times R^{+} \\
\omega(x, 0)=\omega_{0}(x)=a(x) \tilde{v}_{0}(x), \\
\omega(0, t)=\omega_{-} \\
\omega(+\infty, t)=\omega_{+}>0
\end{array}\right.
$$

The first equation of (1.8) is not invariant in the diffusive rescaling. As in [1], we can still hope that the large-time profiles are invariant in the diffusive rescaling for $w(x, t)$. Thus, it is expected that the asymptotic profile of (1.8) can be described by

$$
\left\{\begin{array}{l}
\bar{\omega}_{t}+a_{1}\left(\bar{\omega}^{-\gamma}\right)_{x x}=0, \quad(x, t) \in R^{+} \times R^{+}, \\
\bar{\omega}(0, t)=\omega_{-}, \quad \omega(+\infty, t)=\omega_{+},
\end{array}\right.
$$

where $a_{1}=(\gamma-1) e^{-\frac{1}{\gamma} s_{+}}$. This problem has self-similar solutions $\bar{w}$. Let $\bar{v}=a^{-1} \bar{w}$ and $\bar{u}=$ $-\left(\bar{w}^{-\gamma}\right)_{x}$, using the $L^{1}$ estimates and energy method, Pan [1] proved the global existence and the asymptotic behavior of solutions for (1.5) by comparing $w$ with $\bar{w}$. Our first main theorem is as follows.

Theorem 1.1 Let $\omega_{0}(x)$ and $s_{0}(x)$ be $C^{2}$ functions such that $x\left(s_{0}(x)-s_{+}\right) \in L^{1}\left(R^{+}\right)$and $w_{0}(x)-\bar{w}(x, 0) \in\left(H^{2} \cap L^{1}\right)\left(R^{+}\right)$. There exists a number $\delta_{0}>0$ such that, if $0<\delta \leq \delta_{0}$ and the initial perturbation $\left|\omega_{+}-\omega_{-}\right|+\left\|\omega_{0}(x)-\bar{\omega}(x, 0)\right\|_{H^{2}} \leq \delta$, then the global solution $(\tilde{v}, \tilde{u}, s)(x, t)$ of problem (1.5) exists and satisfies

$$
\omega(x, t)-\bar{\omega} \in C\left([0, t] ; H^{2}\right), \quad \text { for } t>0 .
$$

Moreover, the following convergence rates are true:

$$
\|(\tilde{v}-\bar{v})(\cdot, t)\|_{L^{\infty}} \leq C(1+t)^{-\frac{1}{2}}, \quad\|(\tilde{u}-\bar{u})(\cdot, t)\|_{L^{\infty}} \leq C(1+t)^{-1} .
$$

Remark 1.1 The existence of the global smooth solution for the problem (1.5) has been established by Pan in [1]. Our main contribution is the new convergence rates in (1.10) obtained. These rates are better than that obtained by Pan in [1].

Now, we introduce the auxiliary functions $(\hat{u}, \hat{v})(x, t)$ as in [1],

$$
\left\{\begin{array}{l}
\hat{u}(x, t)=\left[\left(u_{0}(0)-u_{+}\right) \int_{x}^{+\infty} m_{0}(\xi) d \xi+u_{+}\right] e^{-t} \\
\hat{v}(x, t)=\left(u_{0}(0)-u_{+}\right) m_{0}(x) e^{-t}
\end{array}\right.
$$

where $m_{0}(x)$ is a smooth and compact supported function such that

$$
\int_{0}^{+\infty} m_{0}(x) d x=1
$$


Then the function $(\hat{u}, \hat{v})(x, t)$ satisfies

$$
\left\{\begin{array}{l}
\hat{v}_{t}-\hat{u}_{x}=0, \quad(x, t) \in R^{+} \times R^{+} \\
\hat{u}_{t}=-\hat{u} \\
(\hat{v}, \hat{u})(+\infty, t)=\left(0, u_{+} e^{-t}\right) \\
\left(\hat{u}, \hat{v}, \hat{u}_{x}\right)(0, t)=\left(u_{0}(0) e^{-t}, 0,0\right)
\end{array}\right.
$$

Hence, from (1.1), (1.5) and (1.13), we have

$$
\left\{\begin{array}{l}
(v-\tilde{v}-\hat{v})_{t}-(u-\tilde{u}-\hat{u})_{x}=0, \\
(u-\tilde{u}-\hat{u})_{t}+(p(v, s)-p(\tilde{v}, s))_{x}=p(\tilde{v}, s)_{x t}-(u-\tilde{u}-\hat{u}) .
\end{array}\right.
$$

Define

$$
y=-\int_{x}^{+\infty}[v(\xi, t)-\tilde{v}(\xi, t)-\hat{v}(\xi, t)] d \xi
$$

which satisfies the following problem:

$$
\left\{\begin{array}{l}
y_{t t}+\left[p\left(y_{x}+\tilde{v}+\hat{v}, s\right)-p(\tilde{v}, s)\right]_{x}+y_{t}=p(\tilde{v}, s)_{x t}, \quad(x, t) \in R^{+} \times R^{+} \\
y(x, 0)=y_{0}(x)=-\int_{x}^{+\infty}\left[v_{0}(\xi)-\tilde{v}(\xi, 0)-\hat{v}(\xi, 0)\right] d \xi \\
y_{t}(x, 0)=y_{1}(x)=u_{0}(x)-\tilde{u}(x, 0)-\hat{u}(x, 0) \\
y_{x}(0, t)=0
\end{array}\right.
$$

The main result of this paper is as follows.

Theorem 1.2 Let $\left(y_{0}, y_{1}\right) \in H^{3}\left(R^{+}\right) \times H^{2}\left(R^{+}\right)$. There exists a number $\delta_{0}>0$ such that, if $0<\delta<\delta_{0}$ and $\left|v_{+}-v_{-}\right|+\left\|y_{0}\right\|_{H^{3}}+\left\|y_{1}\right\|_{H^{2}} \leq \delta$, then the global smooth solution $y$ for the problem (1.16) uniquely exists and satisfies

$$
\|y(\cdot, t)\|_{H^{3}}^{2}+\left\|y_{t}(\cdot, t)\right\|_{H^{2}}^{2}+\int_{0}^{t}\left\|\left(y_{x}, y_{t}\right)(\cdot, \tau)\right\|_{H^{2}}^{2} d \tau \leq C \delta^{2}
$$

and

$$
\left\|y_{x}(\cdot, t)\right\|_{L^{\infty}} \leq C(1+t)^{-\frac{3}{4}}, \quad\left\|y_{t}(\cdot, t)\right\|_{L^{\infty}} \leq C(1+t)^{-\frac{5}{4}}
$$

Furthermore, $(v, u, s)(x, t)=\left(\tilde{v}+\hat{v}+y_{x}, \tilde{u}+\hat{u}+y_{t}, s\right)$ is the global smooth solution of the problem (1.1)-(1.3) which satisfies

$$
\|(v-\tilde{v})(\cdot, t)\|_{L^{\infty}} \leq C(1+t)^{-\frac{3}{4}}, \quad\|(u-\tilde{u})(\cdot, t)\|_{L^{\infty}} \leq C(1+t)^{-\frac{5}{4}},
$$

and

$$
\|(v-\bar{v})(\cdot, t)\|_{L^{\infty}} \leq C(1+t)^{-\frac{1}{2}}, \quad\|(u-\bar{u})(\cdot, t)\|_{L^{\infty}} \leq C(1+t)^{-1} .
$$


Remark 1.2 In [1], the convergence rates are

$$
\begin{aligned}
& \|(v-\tilde{v})(\cdot, t)\|_{L^{\infty}} \leq C(1+t)^{-\frac{1}{2}}(1+\log (1+t))^{\beta_{1}}, \\
& \|(u-\tilde{u})(\cdot, t)\|_{L^{\infty}} \leq C(1+t)^{-1}(1+\log (1+t))^{\beta_{2}},
\end{aligned}
$$

where $\beta_{1}>\frac{1}{3}, \beta_{2}>\frac{1}{2}$, but, in Theorem 1.2, we obtain the better convergence results (1.19) which do not have $(1+\log (1+t))^{\beta_{1}}$ and $(1+\log (1+t))^{\beta_{2}}$.

This paper is organized as follows. In Sect. 2, by applying the technical time-weighted energy method, we will prove the new decay rates of the solutions for the hyperbolic problem (1.1)-(1.3). In Sect. 3, we will employ the same methods used in Sect. 2 to the problem (1.1), (1.2) and (1.4).

Notations. Hereafter, the symbol $C$ or $O(1)$ will always be used to represent several generic constants which are independent of $x$ and $t . L^{p}=L^{p}\left(R^{+}\right)(1 \leq p \leq \infty)$ denotes Lebesgue space with its norm

$$
\|f\|_{L^{p}}=\left(\int_{0}^{+\infty}|f(x)|^{p} \mathrm{~d} x\right)^{\frac{1}{p}}, \quad 1 \leq p<\infty, \quad \text { and } \quad\|f\|_{L^{\infty}}=\sup _{R^{+}}|f(x)| .
$$

Denote $H^{m}(m \geq 0)$ by the Sobolev space with the norm

$$
\|f\|_{H^{m}}=\left(\sum_{i=0}^{m}\left\|\partial_{x}^{i} f\right\|^{2}\right)^{\frac{1}{2}}, \quad\|\cdot\|=\|\cdot\|_{0}=\|\cdot\|_{L^{2}} .
$$

\section{Convergence to diffusive problem (1.5)}

In this section, we will be concerned with obtaining the better decay rates of the diffusive problem (1.5). Let $\bar{\omega}(\eta)$ (with $\eta=\frac{x}{\sqrt{t+1}}$ ) be the similarity solution of (1.9). From the argument in [1], we see that $\bar{\omega}$ has the following properties.

Lemma 2.1 ([1]) For $i \geq 0, j \geq 0$ and $i+j \geq 1$, one has

$$
\left\{\begin{array}{l}
\left|\bar{\omega}(\eta)-\omega_{+}\right|+\left|\bar{\omega}^{\prime}(\eta)\right|+\left|\bar{\omega}^{\prime \prime}(\eta)\right| \leq C\left|\omega_{+}-\omega_{-}\right| e^{-C_{2} \eta^{2}} \\
\bar{\omega}_{t}=-\frac{1}{2}(1+t)^{-1} \eta \bar{\omega}^{\prime}(\eta), \quad \bar{\omega}_{x}=(1+t)^{-\frac{1}{2}} \bar{\omega}^{\prime}(\eta), \\
\left\|\partial_{t}^{i} \partial_{x}^{j} \bar{\omega}(\cdot, t)\right\| \leq C\left|\omega_{+}-\omega_{-}\right|(1+t)^{-(i+j / 2)+1 / 4} \\
\left\|\partial_{t}^{i} \partial_{x}^{j} \bar{\omega}(\cdot, t)\right\|_{L^{\infty}} \leq C\left|\omega_{+}-\omega_{-}\right|(1+t)^{-(i+j / 2)}
\end{array}\right.
$$

Let $\phi=\omega-\bar{\omega}$, then, from (1.5) and (1.8), we get the equations for $\phi$ as follows:

$$
\left\{\begin{array}{l}
\phi_{t}+a(x)(\psi(\bar{\omega}) \phi)_{x x}+\left(a-a_{1}\right)\left(\bar{\omega}^{-\gamma}\right)_{x x}+a(x)\left(g(\phi, \omega) \phi^{2}\right)_{x x}=0 \\
\phi(x, 0)=\phi_{0}(x)=\omega_{0}-\bar{\omega}(x, 0) \\
\phi(0, t)=0
\end{array}\right.
$$

where we have set

$$
\psi(\bar{\omega})=-\gamma \bar{\omega}^{-(\gamma+1)}, \quad \text { and } \quad g(\phi, \omega) \phi^{2}=(\phi+\bar{\omega})^{-\gamma}-\bar{\omega}^{-\gamma}-\psi(\bar{\omega}) \phi
$$


Let $F=-\psi(\bar{\omega}) \phi$, then we have the problem for $F$ as

$$
\left\{\begin{array}{l}
F_{t}+a(x) \psi(\bar{\omega}) F_{x x}-\psi(\bar{\omega})\left(a-a_{1}\right)\left(\bar{\omega}^{-\gamma}\right)_{x x} \\
\quad-\psi_{1}(\bar{\omega}) F \bar{\omega}_{t}-a \psi(\bar{\omega})\left(f F^{2}\right)_{x x}=0, \quad(x, t) \in R^{+} \times R^{+}, \\
F(x, 0)=F_{0}(x)=-\psi(\bar{\omega}(x, 0)) \phi_{0}(x), \\
F(0, t)=0
\end{array}\right.
$$

where

$$
-\psi_{1}(\bar{\omega}) F=\psi^{\prime}(\bar{\omega}) \phi \quad \text { and } \quad f F^{2}=g \phi^{2} .
$$

The main theorem of this section is as follows.

Theorem 2.1 Let $F_{0}(x)$ and $s(x)=s_{0}(x)$ to be smooth functions such that $x\left(s(x)-s_{+}\right) \in$ $L^{1}\left(R^{+}\right)$, and $F_{0} \in H^{2}\left(R^{+}\right) \cap L^{1}\left(R^{+}\right)$. Then there exist a number $\varepsilon_{1}>0$ and $\delta>0$ such that, if $\left|\omega_{+}-\omega_{-}\right| \leq \delta$ and $\left\|F_{0}\right\|_{H^{2}} \leq \varepsilon_{1}$, then, for any fixed $0<\varepsilon_{0}<\frac{1}{2}$, the global smooth solution $F$ of the problem (2.3) uniquely exists and satisfies

$$
\sum_{k=0}^{2}(1+t)^{k+\frac{1}{2}}\left\|\partial_{x}^{k} F(\cdot, t)\right\|^{2} \leq C
$$

and

$$
\sum_{k=1}^{3} \int_{0}^{t}(1+\tau)^{\varepsilon_{0}+k+\frac{1}{2}}\left\|\partial_{x}^{k} F(\cdot, \tau)\right\|^{2} d \tau \leq C(1+t)^{\varepsilon_{0}}
$$

Remark 2.1 In Theorem 2.1, we obtain the better convergence rates (2.4) which do not have $(1+\log (1+t))^{k}, k>1$, in comparison with that obtained by Pan in [1].

The existence of the global solution for the problem (2.3) has been obtained by Pan in [1]. Our interests are to get the better decay rates which will be completed by the following of lemmas.

Pan [1] has obtained the following $L^{1}$-estimate which will be used in the proof of Theorem 2.1.

Lemma 2.2 ([1]) Under the assumptions of Theorem 2.1, the solutions $\phi$ of the problem (2.2) satisfies

$$
\|\phi(\cdot, t)\|_{L^{1}} \leq C_{2}\left(\left\|\phi_{0}\right\|_{L^{1}}+\delta\right)
$$

By virtue of the counterintuitive method by Nishikawa in [23], and using Lemma 2.2, we can obtain the following convergence estimates on $F$.

Lemma 2.3 For any fixed $0<\varepsilon_{0}<\frac{1}{2}$, the solution $F$ of the problem (2.3) in Theorem 2.1 satisfies

$$
(1+t)^{\frac{1}{2}}\|F(\cdot, t)\|^{2}+(1+t)^{\frac{3}{2}}\left\|F_{x}(\cdot, t)\right\|^{2} \leq C,
$$




$$
\sum_{i=1}^{2} \int_{0}^{t}(1+\tau)^{\frac{1}{2}+i+\varepsilon_{0}}\left\|\partial_{x}^{i} F(\cdot, \tau)\right\|^{2} d \tau \leq C(1+t)^{\varepsilon_{0}}
$$

Proof We multiply Eq. $(2.3)_{1}$ by $a^{-1} F$, then we have

$$
\begin{aligned}
\frac{1}{2} \frac{d}{d t}\left(a^{-1} F \phi\right)+F_{x}^{2}= & -\frac{1}{2} a^{-1} \psi_{2}(\bar{\omega}) \bar{\omega}_{t} F^{2}-a^{-1}\left(a-a_{1}\right)\left(\bar{\omega}^{-\gamma}\right)_{x x} F \\
& -\left(f F^{2}\right)_{x} F_{x}+\{\cdots\}_{x}
\end{aligned}
$$

with $\psi_{2}(\bar{\omega}) F^{2}=\phi^{2} \psi^{\prime}(\bar{\omega})$ and $\{\cdots\}_{x}$ is the term which will vanish after integration with respect to $x$. Integrating $(1+t)^{\varepsilon_{0}+\frac{1}{2}} \times(2.9)$ over $(0,+\infty) \times[0, t]$, we then have

$$
\begin{aligned}
\frac{1}{2}(1+ & t)^{\varepsilon_{0}+\frac{1}{2}} \int_{0}^{+\infty} a^{-1} F \phi d x+\int_{0}^{t} \int_{0}^{+\infty}(1+\tau)^{\varepsilon_{0}+\frac{1}{2}} F_{x}^{2} d x d \tau \\
\leq & \frac{1}{2} \int_{0}^{+\infty} a^{-1} F_{0} \phi_{0} d x+\left|\frac{1}{2}\left(\varepsilon_{0}+\frac{1}{2}\right) \int_{0}^{t} \int_{0}^{+\infty}(1+\tau)^{\varepsilon_{0}-\frac{1}{2}} a^{-1} F \phi d x d \tau\right| \\
& +\left|\frac{1}{2} \int_{0}^{t} \int_{0}^{+\infty}(1+\tau)^{\varepsilon_{0}+\frac{1}{2}} a^{-1} \psi_{2}(\bar{\omega}) \bar{\omega}_{t} F^{2} d x d \tau\right| \\
& +\left|\int_{0}^{t} \int_{0}^{+\infty}(1+\tau)^{\varepsilon_{0}+\frac{1}{2}} a^{-1}\left(a-a_{1}\right)\left(\bar{\omega}^{-\gamma}\right)_{x x} F d x d \tau\right| \\
& +\left|\int_{0}^{t} \int_{0}^{+\infty}(1+\tau)^{\varepsilon_{0}+\frac{1}{2}}\left(f F^{2}\right)_{x} F_{x} d x d \tau\right| \\
\equiv & \frac{1}{2} \int_{0}^{+\infty} a^{-1} F_{0} \phi_{0} d x+\sum_{i=1}^{4} I_{i} .
\end{aligned}
$$

We estimate $I_{1}-I_{4}$ as follows:

$$
\begin{aligned}
I_{1} & I_{2}+I_{3} \\
& \leq C \int_{0}^{t} \int_{0}^{+\infty}\left[(1+\tau)^{\varepsilon_{0}-\frac{1}{2}} F^{2}+(1+\tau)^{\varepsilon_{0}+\frac{1}{2}}\left(\left|\bar{\omega}_{t}\right| F^{2}+\left|\left(a-a_{1}\right) \bar{\omega}_{t} F\right|\right)\right] d x d \tau \\
& \leq C \int_{0}^{t}(1+\tau)^{\varepsilon_{0}-\frac{1}{2}}\left(\|F\|_{L^{\infty}}\|F\|_{L^{1}}+\delta\left\|x\left(s-s_{1}\right)\right\|_{L^{1}}\|F\|_{L^{\infty}}\right) d \tau \\
& \leq C(1+\delta) \int_{0}^{t}(1+\tau)^{\varepsilon_{0}-\frac{1}{2}}\left\|F_{x}\right\|^{\frac{2}{3}} d \tau \\
& \leq\left(\frac{1}{4}+C \delta\right) \int_{0}^{t}(1+\tau)^{\varepsilon_{0}+\frac{1}{2}}\left\|F_{x}\right\|^{2} d \tau+C \int_{0}^{t}(1+\tau)^{\varepsilon_{0}-1} d \tau \\
& \leq\left(\frac{1}{4}+C \delta\right) \int_{0}^{t}(1+\tau)^{\varepsilon_{0}+\frac{1}{2}}\left\|F_{x}\right\|^{2} d \tau+C(1+t)^{\varepsilon_{0}},
\end{aligned}
$$

where in the third inequality, we have used

$$
\|F\|_{L^{\infty}} \leq C\left\|F_{x}\right\|^{\frac{2}{3}}
$$

since

$$
\|F\|_{L^{\infty}} \leq C\|F\|^{\frac{1}{2}}\left\|F_{x}\right\|^{\frac{1}{2}} \leq C\|F\|_{L^{\infty}}^{\frac{1}{4}}\|F\|_{L^{1}}^{\frac{1}{4}}\left\|F_{x}\right\|^{\frac{1}{2}} .
$$




$$
\begin{aligned}
I_{4} & =\left|\int_{0}^{t} \int_{0}^{+\infty}(1+\tau)^{\varepsilon_{0}+\frac{1}{2}}\left(2 f F F_{x}+f_{F} F^{2} F_{x}+f_{\bar{\omega}} \bar{\omega}_{x} F^{2}\right) F_{x} d x d \tau\right| \\
& \leq C \varepsilon_{1} \int_{0}^{t}(1+\tau)^{\varepsilon_{0}+\frac{1}{2}}\left\|F_{x}\right\|^{2} d \tau+C \varepsilon_{1} \int_{0}^{t}(1+\tau)^{\varepsilon_{0}+\frac{1}{2}}\|F\|_{L^{1}}\|F\|_{L^{\infty}}^{3} d \tau \\
& \leq C \varepsilon_{1} \int_{0}^{t}(1+\tau)^{\varepsilon_{0}+\frac{1}{2}}\left\|F_{x}\right\|^{2} d \tau .
\end{aligned}
$$

By using the smallness of $\delta$ and $\varepsilon_{1}$, we have from (2.10)-(2.13)

$$
(1+t)^{\varepsilon_{0}+\frac{1}{2}}\|F(\cdot, t)\|^{2}+\int_{0}^{t}(1+\tau)^{\varepsilon_{0}+\frac{1}{2}}\left\|F_{x}(\cdot, \tau)\right\|^{2} d \tau \leq C(1+t)^{\varepsilon_{0}} .
$$

For second-order energy estimates, let us multiply (2.3) by $-F_{x x}$, it follows that

$$
\begin{aligned}
\frac{1}{2} \frac{d}{d t} F_{x}^{2}-a(x) \psi(\bar{\omega}) F_{x x}^{2}= & -\psi(\bar{\omega})\left(a-a_{1}\right)\left(\bar{\omega}^{-\gamma}\right)_{x x} F_{x x}-\psi_{1}(\bar{\omega}) \bar{\omega}_{t} F F_{x x} \\
& -a(x) \psi(\bar{\omega})\left(f F^{2}\right)_{x x} F_{x x}+\{\cdots\}_{x} .
\end{aligned}
$$

Integrating $(1+t)^{\varepsilon_{0}+\frac{3}{2}} \times(2.15)$ over $[0, t] \times(0,+\infty)$, we have

$$
\begin{aligned}
(1+ & t)^{\varepsilon_{0}+\frac{3}{2}} \int_{0}^{+\infty} F_{x}^{2} d x+\int_{0}^{t} \int_{0}^{+\infty}(1+\tau)^{\varepsilon_{0}+\frac{3}{2}} F_{x x}^{2} d x d \tau \\
\leq & C+C\left(\int_{0}^{t} \int_{0}^{+\infty}(1+\tau)^{\varepsilon_{0}+\frac{1}{2}} F_{x}^{2} d x d \tau+\left|\int_{0}^{t} \int_{0}^{+\infty}(1+\tau)^{\varepsilon_{0}+\frac{3}{2}} F \bar{\omega}_{t} F_{x x} d x d \tau\right|\right. \\
& +\left|\int_{0}^{t} \int_{0}^{+\infty}(1+\tau)^{\varepsilon_{0}+\frac{3}{2}}\left(a-a_{1}\right) \bar{\omega}_{t} F_{x x} d x d \tau\right| \\
& \left.+\left|\int_{0}^{t} \int_{0}^{+\infty}(1+\tau)^{\varepsilon_{0}+\frac{3}{2}}\left(f F^{2}\right)_{x x} F_{x x} d x d \tau\right|\right) .
\end{aligned}
$$

Using Lemma 2.1 and the Cauchy-Schwartz inequality, we have

$$
\begin{aligned}
(1+ & t)^{\varepsilon_{0}+\frac{3}{2}} \int_{0}^{+\infty} F_{x}^{2} d x+\int_{0}^{t} \int_{0}^{+\infty}(1+\tau)^{\varepsilon_{0}+\frac{3}{2}} F_{x x}^{2} d x d \tau \\
\leq & C+C\left(\int_{0}^{t} \int_{0}^{+\infty}(1+\tau)^{\varepsilon_{0}+\frac{3}{2}}\left|F \bar{\omega}_{t}\right|^{2} d x d \tau\right. \\
& +\int_{0}^{t} \int_{0}^{+\infty}(1+\tau)^{\varepsilon_{0}+\frac{3}{2}}\left|\left(a-a_{1}\right) \bar{\omega}_{t}\right|^{2} d x d \tau \\
& \left.+\int_{0}^{t} \int_{0}^{+\infty}(1+\tau)^{\varepsilon_{0}+\frac{3}{2}}\left|\left(f F^{2}\right)_{x x}\right|^{2} d x d \tau\right) .
\end{aligned}
$$

Since

$$
\begin{aligned}
& \int_{0}^{t} \int_{0}^{+\infty}(1+\tau)^{\varepsilon^{\varepsilon^{+}}+\frac{3}{2}}\left|F \bar{\omega}_{t}\right|^{2} d x d \tau \\
& \quad \leq C \int_{0}^{t}(1+\tau)^{\varepsilon_{0}+\frac{3}{2}}\|F\|_{L^{\infty}}\|F\|_{L^{1}}\left\|\bar{\omega}_{t}\right\|_{L^{\infty}}^{2} d \tau \\
& \quad \leq C \int_{0}^{t}(1+\tau)^{\varepsilon_{0}-\frac{1}{2}}\|F\|_{L^{\infty}} d \tau \leq C \int_{0}^{t}(1+\tau)^{\varepsilon_{0}-\frac{1}{2}}\left\|F_{x}\right\|^{\frac{3}{2}} d \tau
\end{aligned}
$$




$$
\begin{aligned}
& \leq C \int_{0}^{t}(1+\tau)^{\varepsilon_{0}+\frac{1}{2}}\left\|F_{x}\right\|^{2} d \tau+C \int_{0}^{t}(1+\tau)^{\varepsilon_{0}-1} d \tau \\
& \leq C(1+t)^{\varepsilon_{0}}, \\
\int_{0}^{t} & \int_{0}^{+\infty}(1+\tau)^{\varepsilon_{0}+\frac{3}{2}}\left|\left(a-a_{1}\right) \bar{\omega}_{t}\right|^{2} d x d \tau \\
& \leq C \int_{0}^{t}(1+\tau)^{\varepsilon_{0}+\frac{3}{2}-3}\left\|x\left(a-a_{1}\right)\right\|_{L^{1}}^{2} d \tau \leq C \int_{0}^{t}(1+\tau)^{\varepsilon_{0}-\frac{3}{2}} d \tau \leq C,
\end{aligned}
$$

and

$$
\begin{aligned}
\left(f F^{2}\right)_{x x}= & \left(2 f F F_{x}+f_{F} F^{2} F_{x}+f_{\bar{\omega}} \bar{\omega}_{x} F^{2}\right)_{x} \\
= & \left(2 f F+f_{F} F^{2}\right) F_{x x}+\left(2 f+4 f_{F} F+f_{F F} F^{2}\right) F_{x}^{2} \\
& +\left(4 f_{\bar{\omega}}+2 f_{F \bar{\omega}} F^{2}\right) F_{x} \bar{\omega}_{x}+\left(f_{\bar{\omega}} \bar{\omega}_{x x}+f_{\bar{\omega} \bar{\omega}} \bar{\omega}^{2}\right) F^{2},
\end{aligned}
$$

it follows that

$$
\begin{aligned}
\int_{0}^{t} & \int_{0}^{+\infty}(1+\tau)^{\varepsilon_{0}+\frac{3}{2}}\left|\left(f F^{2}\right)_{x x}\right|^{2} d x d \tau \\
\leq & C \int_{0}^{t} \int_{0}^{+\infty}(1+\tau)^{\varepsilon_{0}+\frac{3}{2}}\left[\left(F^{2}+F^{4}\right)\left(F_{x x}^{2}+\bar{\omega}_{x}^{2} F_{x}^{2}\right)+F_{x}^{4}+\left(\bar{\omega}_{x x}^{2}+\bar{\omega}_{x}^{4}\right) F^{4}\right] d x d \tau \\
\leq & C \varepsilon_{1} \int_{0}^{t}(1+\tau)^{\varepsilon_{0}+\frac{3}{2}}\left\|F_{x x}\right\|^{2} d \tau+\int_{0}^{t}(1+\tau)^{\varepsilon_{0}+\frac{1}{2}}\left\|F_{x}\right\|^{2} d \tau \\
& +\int_{0}^{t}(1+\tau)^{\varepsilon_{0}+\frac{1}{2}}\|F\|_{L^{\infty}}^{3}\|F\|_{L^{1}} d \tau+\int_{0}^{t}(1+\tau)^{\varepsilon_{0}+\frac{3}{2}}\left\|F_{x}\right\|_{L^{4}}^{4} d \tau \\
\leq & C+C \varepsilon_{1} \int_{0}^{t}(1+\tau)^{\varepsilon_{0}+\frac{3}{2}}\left\|F_{x x}\right\|^{2} d \tau+\int_{0}^{t}(1+\tau)^{\varepsilon_{0}+\frac{3}{2}}\left\|F_{x x}\right\|\left\|F_{x}\right\|^{3} d \tau \\
\leq & C+C\left(\varepsilon_{1}+\varepsilon\right) \int_{0}^{t}(1+\tau)^{\varepsilon_{0}+\frac{3}{2}}\left\|F_{x x}\right\|^{2} d \tau \\
& +C(\varepsilon) \int_{0}^{t}(1+\tau)^{\varepsilon_{0}+\frac{3}{2}}\left\|F_{x}\right\|^{2}\left\|F_{x}\right\|^{2} d \tau .
\end{aligned}
$$

By using the smallness of $\varepsilon$ and $\varepsilon_{1}$, we have from (2.16)-(2.20)

$$
\begin{aligned}
& (1+t)^{\varepsilon_{0}+\frac{3}{2}}\left\|F_{x}(\cdot, t)\right\|^{2}+\int_{0}^{t}(1+\tau)^{\varepsilon_{0}+\frac{3}{2}}\left\|F_{x x}(\cdot, \tau)\right\|^{2} d \tau \\
& \quad \leq C\left[(1+t)^{\varepsilon_{0}}+\int_{0}^{t}(1+\tau)^{\varepsilon_{0}+\frac{3}{2}}\left\|F_{x}(\cdot, \tau)\right\|^{2}\left\|F_{x}(\cdot, \tau)\right\|^{2} d \tau\right] .
\end{aligned}
$$

Therefore, using Gronwall's inequality, we have

$$
(1+t)^{\varepsilon_{0}+\frac{3}{2}}\left\|F_{x}(\cdot, t)\right\|^{2}+\int_{0}^{t}(1+\tau)^{\varepsilon_{0}+\frac{3}{2}}\left\|F_{x x}(\cdot, \tau)\right\|^{2} d \tau \leq C(1+t)^{\varepsilon_{0}} .
$$

Hence, (2.14) and (2.21) finish the proof of this lemma.

From Lemma 2.3, we know that Theorem 2.1 has been proved. Now we show the convergence rates for $F_{t}$ and $F_{x x}$. 
Lemma 2.4 The solution $F$ of the problem (2.3) in Theorem 2.1 satisfies

$$
(1+t)^{\varepsilon_{0}+\frac{5}{2}}\left\|F_{t}(\cdot, t)\right\|^{2}+\int_{0}^{t}(1+\tau)^{\varepsilon_{0}+\frac{5}{2}}\left\|F_{x t}(\cdot, \tau)\right\|^{2} d \tau \leq C(1+t)^{\varepsilon_{0}}
$$

Proof Let us differentiate on (2.3) in $t$ and multiply the result equation by $a^{-1} F_{t}$, then, it follows that

$$
\begin{aligned}
\frac{1}{2} & \frac{d}{d t}\left(a^{-1} F_{t}^{2}\right)-\psi(\bar{\omega}) F_{x t}^{2}+\psi(\bar{\omega})_{x x} F_{t}^{2}+\psi^{\prime}(\bar{\omega}) \bar{\omega}_{t} F_{x x} F_{t} \\
& -a^{-1}\left(\psi(\bar{\omega})\left(a-a_{1}\right)\left(\bar{\omega}^{-\gamma}\right)_{x x}\right)_{t} F_{t}-a^{-1}\left(\psi_{1}(\bar{\omega}) F \bar{\omega}_{t}\right)_{t} F_{t} \\
& -\left(\psi(\bar{\omega})\left(f F^{2}\right)_{x x}\right)_{t} F_{t}+\{\cdots\}_{x}=0 .
\end{aligned}
$$

Integrating $(1+t)^{\varepsilon_{0}+\frac{5}{2}} \times(2.23)$ over $[0, t] \times(-\infty,+\infty)$, we have

$$
\begin{aligned}
\frac{1}{2}(1+ & t)^{\varepsilon_{0}+\frac{5}{2}} \int_{0}^{+\infty} a^{-1} F_{t}^{2} d x-\int_{0}^{t} \int_{0}^{+\infty}(1+\tau)^{\varepsilon_{0}+\frac{5}{2}} \psi(\bar{\omega}) F_{x t}^{2} d x d \tau \\
= & \frac{1}{2} \int_{0}^{+\infty} a^{-1} F_{1}^{2} d x+\frac{1}{2}\left(\varepsilon_{0}+\frac{5}{2}\right) \int_{0}^{t} \int_{0}^{+\infty}(1+\tau)^{\varepsilon_{0}+\frac{3}{2}} a^{-1} F_{t}^{2} d x d \tau \\
& -\frac{1}{2} \int_{0}^{t} \int_{0}^{+\infty}(1+\tau)^{\varepsilon_{0}+\frac{5}{2}} \psi(\bar{\omega})_{x x} F_{t}^{2} d x d \tau \\
& -\int_{0}^{t} \int_{0}^{+\infty}(1+\tau)^{\varepsilon_{0}+\frac{5}{2}} \psi^{\prime}(\bar{\omega}) \bar{\omega}_{t} F_{x x} F_{t} d x d \tau \\
& +\int_{0}^{t} \int_{0}^{+\infty}(1+\tau)^{\varepsilon_{0}+\frac{5}{2}} a^{-1}\left(\psi(\bar{\omega})\left(a-a_{1}\right)\left(\bar{\omega}^{-\gamma}\right)_{x x}\right)_{t} F_{t} d x d \tau \\
& +\int_{0}^{t} \int_{0}^{+\infty}(1+\tau)^{\varepsilon_{0}+\frac{5}{2}} a^{-1}\left(\psi \psi_{1}(\bar{\omega}) F \bar{\omega}_{t}\right)_{t} F_{t} d x d \tau \\
& +\int_{0}^{t} \int_{0}^{+\infty}(1+\tau)^{\varepsilon_{0}+\frac{5}{2}}\left(\psi(\bar{\omega})\left(f F^{2}\right)_{x x}\right)_{t} F_{t} d x d \tau .
\end{aligned}
$$

From the derivation of Lemma 2.3 and $(2.3)_{1}$, we have

$$
\begin{aligned}
\int_{0}^{t}(1+\tau)^{\varepsilon_{0}+\frac{3}{2}}\left\|F_{t}(\cdot, \tau)\right\|^{2} d \tau \\
\leq C\left(\int_{0}^{t}(1+\tau)^{\varepsilon_{0}+\frac{3}{2}}\left\|F_{x x}(\cdot, \tau)\right\|^{2} d \tau+\int_{0}^{t} \int_{0}^{+\infty}(1+\tau)^{\varepsilon_{0}+\frac{3}{2}}\left(a-a_{1}\right)^{2} \bar{\omega}_{t}^{2} d x d \tau\right. \\
\left.\quad+\int_{0}^{t} \int_{0}^{+\infty}(1+\tau)^{\varepsilon_{0}+\frac{3}{2}} F^{2} \bar{\omega}_{t}^{2} d x d \tau+\int_{0}^{t} \int_{0}^{+\infty}(1+\tau)^{\varepsilon_{0}+\frac{3}{2}}\left(f F^{2}\right)_{x x}^{2} d x d \tau\right) \\
\leq C .
\end{aligned}
$$

Moreover one has

$$
\begin{aligned}
& a^{-1}\left(\psi_{1}(\bar{\omega}) F \bar{\omega}_{t}\right)_{t} F_{t}=O(1)\left[\bar{\omega}_{t} F_{t}^{2}+\left(\bar{\omega}_{t}^{2}+\bar{\omega}_{t t}\right) F F_{t}\right], \\
& a^{-1}\left(\psi(\bar{\omega})\left(a-a_{1}\right)\left(\bar{\omega}^{-\gamma}\right)_{x x}\right)_{t} F_{t}=O(1)\left(a-a_{1}\right)\left(\bar{\omega}_{t}^{2}+\bar{\omega}_{t t}\right) F_{t}, \\
& \left(\psi(\bar{\omega})\left(f F^{2}\right)_{x x}\right)_{t} F_{t}=O(1) \bar{\omega}_{t}\left(f F^{2}\right)_{x x} F_{t}-\psi(\bar{\omega})\left(f F^{2}\right)_{x x t} F_{t} .
\end{aligned}
$$


Now, we can employ the similar argument as used in deducing (2.14) to conclude

$$
(1+t)^{\varepsilon_{0}+\frac{5}{2}}\left\|F_{t}(\cdot, t)\right\|^{2}+\int_{0}^{t}(1+\tau)^{\varepsilon_{0}+\frac{3}{2}}\left\|F_{x t}(\cdot, \tau)\right\|^{2} d \tau \leq C(1+t)^{\varepsilon_{0}},
$$

which finishes the proof.

Corollary 2.1 The solution F of the problem (2.3) satisfies

$$
(1+t)^{\frac{5}{2}}\left\|F_{x x}(\cdot, t)\right\|^{2} \leq C, \quad\left\|F_{x}(\cdot, t)\right\|_{L^{\infty}} \leq C(1+t)^{-1} .
$$

Proof From (2.3), we have

$$
F_{x x}=O(1)\left[F_{t}+\left(a-a_{1}\right) \bar{\omega}_{t}+F_{x}^{2}+F_{t} \bar{\omega}_{t}+F F_{x} \bar{\omega}_{x}+\left(\bar{\omega}_{x x}+\bar{\omega}_{x}^{2}\right) F^{2}\right] .
$$

By taking $L^{2}$ norm in (2.28), one has

$$
\begin{aligned}
(1+t)^{\frac{5}{2}}\left\|F_{x x}\right\|^{2} \leq & C(1+t)^{\frac{5}{2}}\left(\left\|F_{t}\right\|^{2}+\left\|F_{x}^{2}\right\|^{2}+\left\|\left(a-a_{1}\right) \bar{\omega}_{t}\right\|^{2}+\left\|F \bar{\omega}_{t}\right\|^{2}\right. \\
& \left.+\left\|F F_{x} \bar{\omega}_{x}\right\|^{2}+\left\|\left(\bar{\omega}_{x x}+\bar{\omega}_{x}^{2}\right) F^{2}\right\|^{2}\right) \\
\leq & C+C(1+t)^{\frac{5}{2}}\left\|F_{x}^{2}\right\|^{2} \\
\leq & C+C(1+t)^{\frac{5}{2}}\left\|F_{x}\right\|^{2}\left(\left\|F_{x}\right\|^{2}+\left\|F_{x x}\right\|^{2}\right) \\
\leq & C+C(1+t)^{\frac{5}{2}}\left\|F_{x}\right\|^{2}\left\|F_{x x}\right\|^{2}
\end{aligned}
$$

which implies

$$
(1+t)^{\frac{5}{2}}\left\|F_{x x}\right\|^{2} \leq C .
$$

Then

$$
\left\|F_{x}\right\|_{L^{\infty}} \leq C\left\|F_{x}\right\|^{\frac{1}{2}}\left\|F_{x x}\right\|^{\frac{1}{2}}(1+t)^{-1} .
$$

This ends the proof of Corollary 2.1.

Setting $\tilde{v}=a^{-1}(x) \omega, \tilde{u}=-\left(\omega^{-\gamma}\right)_{x}$, by using (2.12) and the interpolation inequality, we have the convergence estimate (1.10). Theorem 1.1 then follows from Theorem 2.1.

\section{Convergence rates to the stationary solution}

In this section, we will employ the same technique as that used in Sect. 2 to treat the problem (1.1), (1.2) and (1.4).

Similar to (1.8) in Sect. 2, by using the variables $\omega$ and $a(x)$, the problem (1.6) can be reformulated as

$$
\left\{\begin{array}{l}
\omega_{t}+a(x)\left(\omega^{-\gamma}\right)_{x x}=0, \quad(x, t) \in R^{+} \times R^{+}, \\
\omega(x, 0)=\omega_{0}(x) \rightarrow \omega_{+}, \quad \text { as } x \rightarrow+\infty \\
\omega_{x}(0, t)=0
\end{array}\right.
$$


Setting $\phi=\omega-\omega_{+}$, we get

$$
\left\{\begin{array}{l}
\phi_{t}+a(x) \phi_{x x}+a(x)\left(f_{1}(\phi) \phi^{2}\right)_{x x}=0, \quad(x, t) \in R^{+} \times R^{+} \\
\phi(x, 0)=\phi_{0}(x)=\omega_{0}(x)-\omega_{+} \\
\phi_{x}(0, t)=0
\end{array}\right.
$$

where $f_{1}(\phi) \phi^{2}=\left(\omega_{+}+\phi\right)^{-\gamma}-\omega_{+}^{-\gamma}-b \phi$ and $b=\gamma \omega_{+}^{-\gamma-1}$. Note that if $\phi_{0}(x) \in L^{1}$, then by using the same arguments as that in Lemma 2.2, we have

$$
\|\phi(\cdot, t)\|_{L^{1}} \leq C\left\|\phi_{0}\right\|_{L^{1}}
$$

By using the same method as that in Sect. 2, we can obtain the following result.

Theorem 3.1 Let $\phi_{0}(x)$ and $s_{0}(x)$ to be smooth functions such that $\phi_{0} \in\left(H^{2} \cap L^{1}\right)\left(R^{+}\right)$. There exists a number $\delta_{0}>0$ such that, if $0<\delta \leq \delta_{0}$ and $\left\|\phi_{0}\right\|_{H^{2}} \leq \delta$, then the global smooth solution $\phi(x, t)$ of (3.2) uniquely exists and satisfies

$$
\sum_{i=0}^{2}(1+t)^{i+\frac{1}{2}}\left\|\partial_{x}^{i} \phi(\cdot, \tau)\right\|^{2}+\sum_{i=1}^{3} \int_{0}^{t}(1+\tau)^{i-\frac{1}{2}}\left\|\partial_{x}^{i} \phi(\cdot, \tau)\right\|^{2} d \tau \leq C
$$

By defining $v_{1}=a^{-1} \omega(x, t)=a^{-1}\left(\omega_{+}+\phi(x, t)\right), u_{1}(x, t)=-p\left(v_{1}, s\right)_{x}$, and $v_{*}=a^{-1} \omega_{+}$, then, $\left(v_{1}, u_{1}, s\right)$ is the global solution for problem (1.6) which satisfies

$$
\left\|\left(v_{1}-v_{*}\right)(\cdot, t)\right\|_{L^{\infty}} \leq C(1+t)^{-\frac{1}{2}}, \quad\left\|u_{1}(\cdot, t)\right\|_{L^{\infty}} \leq C(1+t)^{-1} .
$$

Remark 3.1 The new convergence rates in (3.4) obtained are better than that obtained in [1].

Defining the following auxiliary functions $\left(\hat{u}_{1}, \hat{v}_{1}\right)$ :

$$
\left(\hat{u}_{1}, \hat{v}_{1}\right)(x, t)=\left(u_{+} e^{-t} \int_{0}^{x} m_{0}(\xi) d \xi, u_{+} m_{0}(x) e^{-t}\right)
$$

where the smooth function $m_{0}(x)$ is the same as in (1.12). Setting $\tilde{y}=-\int_{x}^{+\infty}\left(v-v_{1}-\right.$ $\left.\hat{v}_{1}\right)(\xi, t) d \xi$, we then have

$$
\left\{\begin{array}{l}
\tilde{y}_{t t}+\left[p\left(\tilde{y}_{x}+v_{1}+\hat{v}_{1}, s\right)-p\left(v_{1}, s\right)\right]_{x}+\tilde{y}_{t}=p\left(v_{1}, s\right)_{x t}, \quad(x, t) \in R^{+} \times R^{+} \\
\tilde{y}(x, 0)=\tilde{y}_{0}(x)=-\int_{x}^{+\infty}\left[v_{0}(\xi)-v_{1}(\xi, 0)-\hat{v}_{1}(\xi, 0)\right] d \xi \\
\tilde{y}_{t}(x, 0)=\tilde{y}_{1}(x)=u_{0}(x)-u_{1}(x, 0)-\hat{u}_{1}(x, 0) \\
\tilde{y}(0, t)=0
\end{array}\right.
$$

Thus, we can also obtain the following result.

Theorem 3.2 There exists a number $\delta_{0}>0$ such that, if $0<\delta \leq \delta_{0}$ and $\left\|\tilde{y}_{0}\right\|_{H^{3}}+\left\|\tilde{y}_{1}\right\|_{H^{2}} \leq$ $\delta^{2}$, then the global smooth solution $(v, u, s)(x, t)$ of the problem (1.1), (1.2) and (1.4) uniquely 
exists and satisfies

$$
\left\|\left(v-v_{1}\right)(\cdot, t)\right\|_{L^{\infty}} \leq C(1+t)^{-\frac{3}{4}}, \quad\left\|\left(u-u_{1}\right)(\cdot, t)\right\|_{L^{\infty}} \leq C(1+t)^{-\frac{5}{4}},
$$

and

$$
\|(v-\bar{v})(\cdot, t)\|_{L^{\infty}} \leq C(1+t)^{-\frac{1}{2}}, \quad\|u(\cdot, t)\|_{L^{\infty}} \leq C(1+t)^{-1} .
$$

Remark 3.2 In [1], the convergence rates are

$$
\begin{aligned}
& \left\|\left(v-v_{1}\right)(\cdot, t)\right\|_{L^{\infty}} \leq C(1+t)^{-\frac{1}{2}}(1+\log (1+t))^{\beta_{1}}, \\
& \|u(\cdot, t)\|_{L^{\infty}} \leq C(1+t)^{-1}(1+\log (1+t))^{\beta_{2}}
\end{aligned}
$$

where $\beta_{1}>\frac{1}{3}, \beta_{2}>\frac{1}{2}$, but, in Theorem 3.2, we obtain the better result (3.8) which does not have $(1+\log (1+t))^{\beta_{1}}$ and $(1+\log (1+t))^{\beta_{2}}$.

\section{Acknowledgements}

Not applicable.

\section{Funding}

The research of L.-N. Zhang was supported by in part by the National Natural Science Foundation of China (No. 11701489), Natural Science Foundation of Hunan Province of China (No. 2018JJ3481). The research of S.-F. Geng was supported in part by Natural Science Foundation of Hunan Province of China (No. 2018JJ2373), Excellent Youth Project of Hunan Education Department (No. 18B054).

\section{Abbreviations}

Not applicable.

Availability of data and materials

Not applicable.

\section{Competing interests}

The authors declare that they have no competing interests.

\section{Authors' contributions}

All the authors contributed to each part of this study equally and approved the final version of the manuscript.

\section{Publisher's Note}

Springer Nature remains neutral with regard to jurisdictional claims in published maps and institutional affiliations.

Received: 8 July 2019 Accepted: 6 December 2019 Published online: 13 December 2019

\section{References}

1. Pan, R.: Boundary effects and large time behavior for the system of compressible adiabatic flow through porous media. Mich. Math. J. 49, 519-540 (2001)

2. Zheng, Y.: Global smooth solution to the adiabatic gas dynamics system with dissipation terms. Chin. Ann. Math. 17A 155-162 (1996)

3. Hsiao, L., Luo, T.: Nonlinear diffusive phenomena of solutions for the system of compressible adiabatic flow through porous media. J. Differ. Equ. 125, 329-365 (1996)

4. Hsiao, L., Serre, D.: Large-time behavior of solutions for the system of compressible adiabatic flow through porous media. Chin. Ann. Math., Ser. B 16, 431-444 (1995)

5. Marcati, P., Pan, R.: On the diffusive profiles for the system of compressible adiabatic flow through porous media. SIAM J. Math. Anal. 33, 790-826 (2001)

6. Nishihara, K., Nishikawa, M.: Asymptotic behavior of solutions to the system of compressible adiabatic flow through porous media. SIAM J. Math. Anal. 33, 216-239 (2001)

7. Geng, S., Wang, Z.: Convergence rates to nonlinear diffusion waves for solutions to the system of compressible adiabatic flow through porous media. Commun. Partial Differ. Equ. 36, 850-872 (2011)

8. Pan, R.: Darcy's law as long-time limit of adiabatic porous media flow. J. Differ. Equ. 220, 121-146 (2006)

9. Hsiao, L., Pan, R.: Initial boundary value problem for the system of compressible adiabatic flow through porous media. J. Differ. Equ. 159, 280-305 (1999) 
10. Hsiao, L., Liu, T.-P.: Convergence to nonlinear diffusion waves for solutions of a system of hyperbolic conservation laws with damping. Commun. Math. Phys. 143, 599-605 (1992)

11. Mei, M.: Best asymptotic profile for hyperbolic $p$-system with damping. SIAM J. Math. Anal. 42, 1-23 (2010)

12. Nishihara, K.: Convergence rates to nonlinear diffusion waves for solutions of system of hyperbolic conservation laws with damping. J. Differ. Equ. 131, 171-188 (1996)

13. Nishihara, K., Wang, W., Yang, T.: $L^{p}$-convergence rate to nonlinear diffusion waves for $p$-system with damping. J. Differ. Equ. 161, 191-218 (2000)

14. Hsiao, L., Liu, T.-P.: Nonlinear diffusion phenomena of nonlinear hyperbolic system. Chin. Ann. Math., Ser. B 14, 465-480 (1993)

15. Huang, F.-M., Mei, M., Wang, Y.: Large time behavior of solutions to n-dimensional bipolar hydrodynamic models for semiconductors. SIAM J. Math. Anal. 43, 1595-1630 (2011)

16. Huang, F.-M., Mei, M., Wang, Y., Yang, T.: Long-time behavior of solutions for bipolar hydrodynamic model of semiconductors with boundary effects. SIAM J. Math. Anal. 44, 1134-1164 (2012)

17. Ma, H., Mei, M.: Best asymptotic profile for linear damped $p$-system with boundary effect. J. Differ. Equ. 249, 446-484 (2010)

18. Marcati, P., Mei, M., Rubino, B.: Optimal convergence rates to diffusion waves for solutions of the hyperbolic conservation laws with damping. J. Math. Fluid Mech. 7, S224-S240 (2005)

19. Wang, Y., Wang, K.: Asymptotic behavior of classical solutions to the compressible Navier-Stokes-Poisson equations in three and higher dimensions. J. Differ. Equ. 259, 25-47 (2015)

20. Xiao, Q., Zhao, H.: Nonlinear stability of planar stationary waves for generalized Benjamin-Bona-Mahony-Burgers equations in the half-plane. J. Hyperbolic Differ. Equ. 10, 283-333 (2013)

21. Zhao, H.: Convergence to strong nonlinear diffusion waves for solutions of $p$-system with damping. J. Differ. Equ. 174, 200-236 (2001)

22. Zhu, C: Convergence rates to nonlinear diffusion waves for weak solutions to $p$-system with damping. Sci. China Ser. A 46, 562-575 (2003)

23. Nishikawa, M.: Convergence rate to the traveling wave for viscous conservation laws. Funkc. Ekvacioj 41, 107-132 (1998)

\section{Submit your manuscript to a SpringerOpen ${ }^{\circ}$ journal and benefit from:}

- Convenient online submission

- Rigorous peer review

- Open access: articles freely available online

- High visibility within the field

- Retaining the copyright to your article

Submit your next manuscript at $\boldsymbol{\Delta}$ springeropen.com 